VIII ${ }^{\text {èmes }}$ Journées Nationales Génie Civil - Génie Côtier, Compiègne, 7-9 septembre 2004

\title{
Analyse de risque pour les ouvrages portuaires: cas de la perforation par corrosion d'un quai gabions
}

\author{
Franck Schoefs $^{(\mathrm{a})}$, Bruno Capra ${ }^{(\mathrm{b})}$, Samuel Masson ${ }^{(\mathrm{c})}$ \\ (a)Maître de conférences, Institut de Recherche en Génie Civil et Mécanique, \\ UMR 6183, Faculté des Sciences de Nantes, BP 92208 - 44322 Nantes cedex 3, \\ 02511255 22-24, schoefs@physique.univ-nantes.fr \\ (b)Directeur scientifique, Société Oxand, 36 bis avenue Franklin Roosevelt, \\ 77210 Avon/Fontainebleau, 33 (0)1 603952 51-bruno.capra@oxand.com \\ (c) GTMa,INSA de Rennes, 20 avenue des buttes de Coësmes, \\ 35043 Rennes cedex, samuel.masson@insa-rennes.fr
}

\section{Résumé:}

La requalification des structures portuaires amène à étudier des ouvrages au comportement incertain intégrant des variabilités importantes. Ces hypothèses diffèrent de celles qui ont présidé à son dimensionnement. Les analyses de risque permettent de sélectionner les états limites fondamentaux d'un point de vue réglementaire et de gestion des ouvrages. Cet article illustre une telle analyse sur un quai gabions soumis à de la corrosion.

\begin{abstract}
:
Reassessment of existing structures leads to investigate uncertainties and randomness in structural behaviour and integrity. Risk screening and analysis allow to take into account these uncertainties and to select the dominant limit state including the requirements of decision maker. This paper illustrates such an analysis on a gabions type wharf submitted to corrosion process.
\end{abstract}

Mots-clés: analyse de risque, corrosion, quai gabions, structures portuaires.

Keywords : risk analysis, wharf, gabions, corrosion.

\section{Introduction}

Les structures portuaires de type quai sont soumises à des conditions environnementales sévères. Les chargements cycliques dus à l'exploitation (portiques, stockage - déstockage, ...) ou à l'environnement sont sources respectivement de dommages de fatigue ou de phénomène de départ des fines des remblais d'arrière quai. La présence d'eaux comportant des teneurs en sels variables, des micro-organismes et des traces de pollution génèrent des corrosions aux cinétiques difficilement prévisibles. Dans la gestion d'un parc d'ouvrages soumis à de tels effets, les gestionnaires doivent disposer d'outils d'aide à la décision permettant de hiérarchiser les travaux en fonction de priorités liées à la sécurité des personnels (présence d'un portique) et à l'exploitation commerciale (niveau stratégique du quai dans le dispositif du port). Par ailleurs, afin d'optimiser ces travaux dans leur efficacité et leur coût, il est nécessaire que ces outils soient prédictifs.

Les analyses de risque présentées dans cet article peuvent constituer le socle d'outils d'aide à la décision. Elles nécessitent de coupler les probabilités de défaillance d'une structure avec les conséquences de ces défaillances. Cet article présente les enjeux d'une analyse de risque dans le cas des quais en général et les illustre sur un quai de type gabion en procédant à 
une sélection des défaillances puis à une évaluation des probabilités associées en cas de corrosion.

\section{$\underline{\text { 2.Evaluation des risques et des incertitudes: cas des structures portuaires }}$}

\subsection{Sélection des risques}

Lorsqu'un gestionnaire étudie la maintenance de kilomètres de quais dont l'age varie de plus de cent ans à quelques années, il est confronté à des méthodes de fabrication, de conception et à des matériaux particulièrement hétérogènes. Il est courant de voir des ouvrages en maçonnerie côtoyer des rideaux de palplanches et des ouvrages en béton armé sur des estacades, des remblais ou des pieux. Il est illusoire de considérer que cette variété peut être analysée au travers de quelques indicateurs d'état issus des inspections. Afin de procéder à une analyse de défaillance il est nécessaire de conduire à une étude globale de l'ouvrage. Cette démarche est délicate puisqu'elle suppose d'analyser l'ouvrage dans son comportement réel diffèrent de celui intégré dans la note de calcul. En particulier, la plupart des règlements de calcul, lorsqu'ils existaient, préconisaient une conception par composant et descente de charge. C’est le cas encore de la majorité des Euronormes dont seuls de rares (Eurocodes 3) ouvrent la possibilité d'utiliser l'hyperstaticité de la structure en cas de défaillance d'un composant (redistribution des efforts après plastification).

La sélection des risques passe par un recensement des défaillances engendrant des conséquences directes jugées inacceptables par le gestionnaire c’est-à-dire nécessitant des travaux et donc des coûts supérieurs à ceux inclus dans le dispositif de maintenance programmée. Par exemple, le tassement ou le déplacement trop important d'un quai peut être admissible au sens de son intégrité mais inacceptable au sens de son exploitation à cause du chemin de portique. La conséquence indirecte chiffrable est l'arrêt d'exploitation et le coût des travaux de confortement. En général, les états limites présents dans les règlements intègrent cette exigence de déplacement de manière masquée et conservative (calcul en sollicitation/résistance) et dans le cas d'une ré-analyse, il doit être spécifié en temps que tel. La figure 1 représente la sélection des calculs d'un ouvrage de type quai gabions à arcs doubles cloisonnés, en fonction du temps. Ce quai est schématisé en figure 2. Il s'agit d'un quai en béton armé sur pieux acier fichés à l'intérieur d'un gabion. Les caractéristiques géométriques (cellules de 30,78 m) et chimiques (salinité de 26,4 g/l et teneur en chlore de 13,97 g/l) sont mentionnées. Pour les ouvrages anciens, trois phases peuvent être distinguées :

- la conception réalisée en général à partir de codes aux contraintes admissibles qui présente le contexte dans lequel l'ouvrage a été conçu (hypothèses, mode de comportement prévu). Dans le cas où les pieux traversent tout le gabion, le monolithisme n’est pas à vérifier.

- la requalification suite à des désordres constatés ou à de nouvelles exigences d'exploitation incompatibles avec les hypothèses initiales. Entre temps, de nouveaux règlements sont parus (codes aux états limites) permettant d'aborder de manière plus fine le comportement de l'ouvrage et les moyens de calculs sont aussi plus performants.

- Quelques temps plus tard, le gestionnaire, fort de cette expérience, peut être amené à prescrire des exigences spécifiques permettant d'anticiper des dysfonctionnements sérieux.

Les enquêtes du type de celle réalisée par Y. Lacroix (voir tableau 1) sont alors précieuses afin de répertorier sur un nombre d'ouvrages significatif les causes de défaillance les plus fréquentes. 


\begin{tabular}{|c|c|c|}
\hline - 20 ans & -10 ans & 'hui \\
\hline $\begin{array}{c}\text { Conception } \\
\text { (normes et DTU) }\end{array}$ & $\begin{array}{c}\text { Requalification : évolution } \\
\text { réglementation et hypothèses } \\
\text { + puissance de calcul }\end{array}$ & $\begin{array}{c}\text { Exigences } \\
\text { du } \\
\text { gestionnaire }\end{array}$ \\
\hline 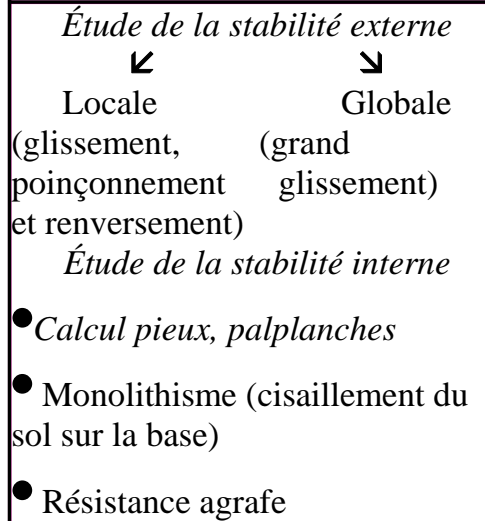 & $\begin{array}{l}\text { Étude de la stabilité externe } \\
\text { (codes) } \\
\text { Étude de la stabilité interne } \\
\text { - Résistance agrafes } \\
\text { - Calcul des pieux et } \\
\text { palplanches (butée / poussée) }\end{array}$ & $\begin{array}{l}\text { Constats des désordres } \\
\text { - Corrosion - perforation } \\
\text { Exigenlacements latéraux } \\
\text { Tassements } \\
\text { Modification hypothèses } \\
\text { Influences des pathologies }\end{array}$ \\
\hline
\end{tabular}

Figure 1: Sélection des défaillances pour un quai gabion.

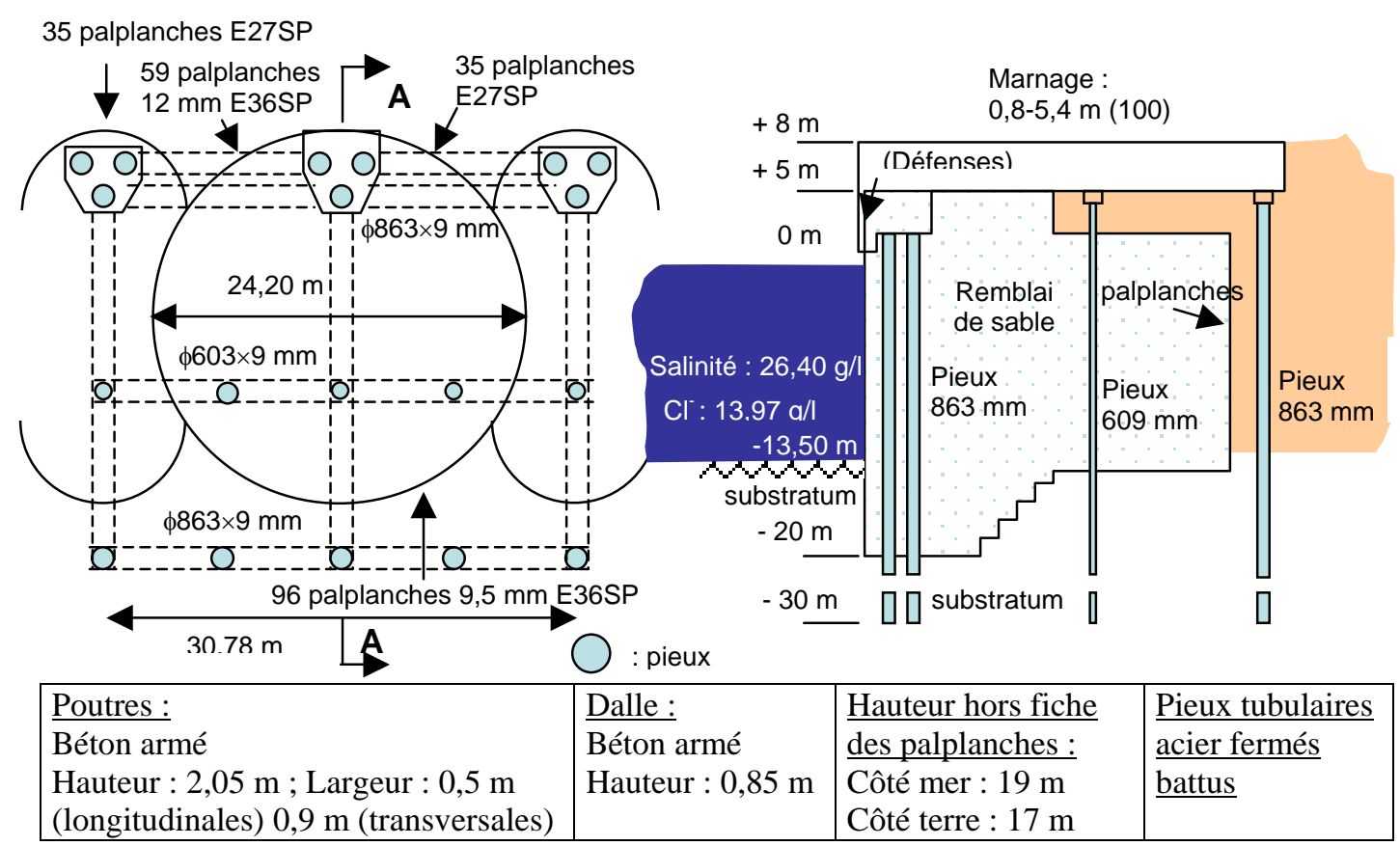

Figure 2: Caractéristiques du quai étudié. 
Tableau 1. Enquête de défaillances sur des quais Gabion, d'après Lacroix.

\begin{tabular}{|l|l|}
\hline \multicolumn{1}{|c|}{ MODE DE RUPTURE } & NOMBRE \\
\hline & 1 \\
Glissement sur la base & 1 \\
Glissement dans le rocher sous la base & \\
Rupture au niveau des palplanches prin- & Plusieurs \\
cipalement aux raccordements & plusieurs \\
Cisaillement du remblai limoneux ou & 1 \\
argileux & \\
"Flottement" d'une cellule & Plusieurs \\
Affouillement et vidange partielle de la & 1 \\
Rupture d'un joint déficient & 2 \\
Battage hors d'un joint & 1 \\
Assemblage'de palplanches inégales & 1 \\
Enlevement prématuré du gabarit & 1 \\
Choc d'une barge & \\
\hline
\end{tabular}

\subsection{Sources d'incertitude pour l'analyse de risque d'un quai gabions.}

Les variables aléatoires prises en compte dans une analyse de risque peuvent être classées en deux catégories ${ }^{1}$ : les aléas dits intrinsèques renvoient à des phénomènes naturels présentant par nature une structure aléatoire ou stochastique et les incertitudes sur les modèles qui intègrent la méconnaissance de certains mécanismes par l'utilisation de modèles simples ou par des erreurs de mesure significatives. Dans le cas des quais gabions on retrouve cette distinction.

D’une part, les aléas intrinsèques sont présents dans :

- le phénomène de corrosion, généré par des eaux dont la composition fluctue en fonction du temps est un champ stochastique c'est-à-dire un champ aléatoire variant en fonction de la position du point de la structure et du temps ,

- l'exploitation du quai (type de navires, nombre et conditions d'accostages),

- le niveau d'eau dû à la marée notamment en cas de sur-cotes². Dans l'illustration présentée en section 2.3, ce phénomène n’apparaît pas et n'est pas intégré. La marée est donc considérée comme pseudo-périodique et déterministe au regard des autres variabilités,

- la caractérisation du remblai après plusieurs années de tassements dus accentués par les modifications cycliques de la charge hydraulique (marée),

- les efforts horizontaux dus aux effets du vent sur les navires et les portiques. Ici, seul l'effet sur les navires est pris en compte.

D’autre part, les aléas sur les modèles sont présents dans :

- le modèle de poussée sur une palplanche dans le cas d'un quai gabions,

- le modèle d'écoulement des constituants du remblai dans le cas d'une perforation de la palplanche.

En cas de perforation par corrosion, on peut assister à une vidange du gabion qui génère une perte de capacité sous chargement latéral (augmentation du déplacement horizontal) et sous chargement vertical (perte de capacité de frottement latéral). Les capacités du sol ont été modélisées sous forme de variables aléatoires à l'aide d'essais au pressiomètre. Les variables de chargements dus aux navires proviennent de la modélisation des statistiques de fréquentation du quai.

Les statistiques de vent permettent quant à elles de modéliser le chargement du portique. Le critère de gestion était du type exploitation avec une borne indiquée par le gestionnaire en terme de déplacement de $1 \mathrm{~cm}$. On a montré ${ }^{3}$ que les probabilités de défaillance associées sont négligeables (probabilité inférieure à $10^{-6}$ ) dans le cas étudié. 
Une autre question a été abordée. Elle concerne la concurrence temporelle des deux phénomènes présents; l'augmentation de la perforation par corrosion (vitesse de 0.023 $\mathrm{mm} / \mathrm{an}$ ) et le mécanisme de vidange. Pour des perforations semi-infinies de 10 et $20 \mathrm{~cm}$ de hauteur une pré-étude a été réalisée avec les logiciels $\mathrm{PGC}^{2 \mathrm{D}} 3.00^{4}$ et Plaxis ${ }^{5}$, par la simulation d'un écoulement granulaire en deux dimensions. Le sol est un sable dont la dispersion granulaire est incluse dans l'intervalle [0.5-5]. L'angle de frottement macroscopique ${ }^{6,7} \mathrm{du}$ remblai est supposé de $30^{\circ}$ avec une cohésion nulle et ce qui correspond à un frottement microscopique à introduire dans les logiciels de $45^{\circ}$. Ceci permet d'intégrer un effet de forme non pris en compte par ailleurs (grains de forme circulaire). Le poids volumique du sol humide et sec est respectivement de $20 \mathrm{kN} / \mathrm{m}^{3}$ et $16 \mathrm{kN} / \mathrm{m}^{3}$. La zone simulée est présentée à la figure 3 et correspond à une zone carré de $45 \mathrm{~cm}$ de côté afin de se placer à une échelle suffisante par rapport aux grains sans engendrer des temps de calcul trop longs (inférieurs à 2 jours). Les conditions aux limites ont été fixées de manière à représenter le massif environnant (poids) ainsi que le frottement des grains sur les parois et le long de la perforation. Le gradient hydraulique correspondant à une pleine mer moyenne a été introduit et la cinématique présentée en figure 3 a été obtenue. Les débits obtenus avoisinent alors 0,1 $\mathrm{m}^{3} / \mathrm{mn}$ pour une perforation de $10 \mathrm{~cm} \times 10 \mathrm{~cm}$. Des études complémentaires sont nécessaires en laboratoire car les cinétiques de vidange et de corrosion ne sont pas du même ordre de grandeur. Seule la cinétique de corrosion uniforme est prise en compte par la suite.

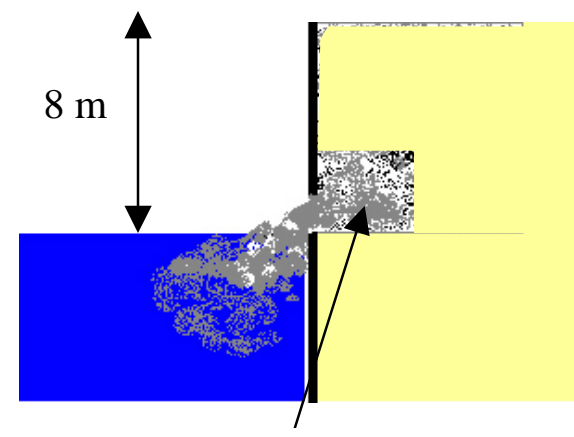

Zone simulée : $0.45 \mathrm{~m}$ x $0.45 \mathrm{~m}$

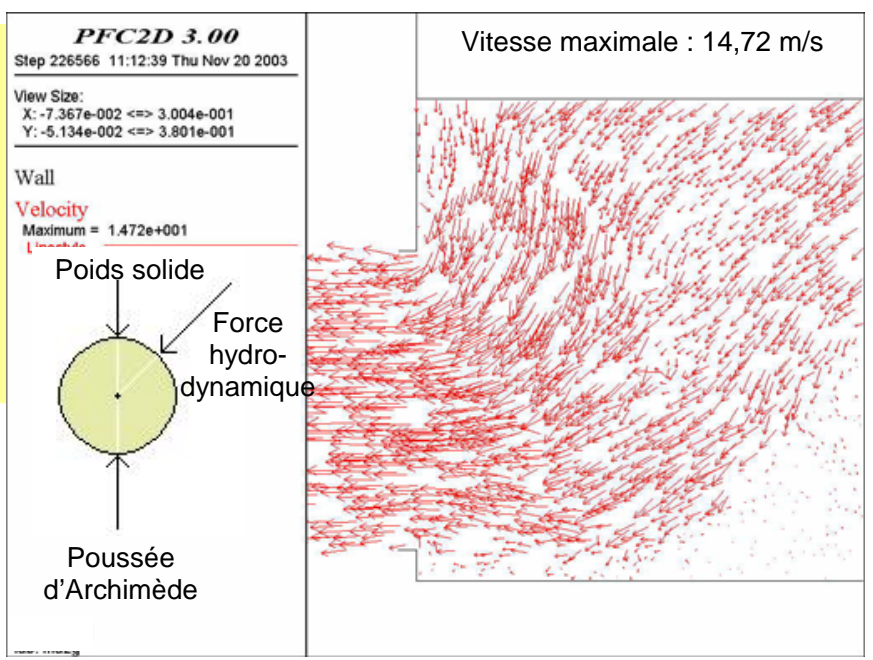

Figure 3:Simulation d'un écoulement par une perforation

\subsection{Modélisation des sources d'incertitude sur la corrosion}

Les cinétiques de corrosion électrochimique et bactériologique restent encore méconnues même si de nombreuses études ont été réalisées ${ }^{8,9,10,11}$. On montre en particulier que les zones de forte corrosion sont situées aux interfaces (zone de marnage, interface sol-eau). La corrosion s'installe pendant les six premiers mois où la vitesse peut être jusqu'à 10 fois supérieure à celle des années suivantes. On considère que durant cette période d'initiation, la sur-épaisseur d'acier à la conception (3 mm) a été consommée. Ensuite, le choix s'est porté sur une cinétique décroissante en fonction du temps (voir figure 4). Pour la cote $0 \mathrm{~m}$ Cartes Marines, on trouve:

$\operatorname{Vcor}(\mathrm{t})_{0}=0,48-0,0231 \mathrm{t}(\mathrm{t}=0: 6$ mois après installation $)$ 
A 6 mois ( $\mathrm{t}=0$ pour le modèle d'évolution probabiliste), ce champ stochastique est une variable aléatoire suivant une loi normale de coefficient de variation $C O V_{t=0}=12 \%$. Ce point est abordé plus en détails dans la litérature ${ }^{12}$. Cette valeur est calculée sur le principe que l'étendue des valeurs fournies par les experts est inclue dans l'intervalle $[\mu-3 \sigma ; \mu+3 \sigma]$ avec $\sigma$, écart-type et $\mu$, moyenne. On trouve $\mu=0,125 \mathrm{~mm} / \mathrm{an}$ et $\sigma=0,015 \mathrm{~mm} / \mathrm{an}$ d'où $C O V_{t=0}=12 \%$. L'écart type est supposé croître linéairement en fonction du temps (capacité prédictive du modèle) ; le coefficient de variation est choisi tel que les données à $\mathrm{t}=0$ constituent la borne supérieure des vitesses de corrosion. Le quantile à $(-3 \sigma)$ est alors supposé suivre une évolution constante correspondant à ce quantile à 6 mois. On obtient alors :

$$
\sigma(t)=0,0576+(0,0231 / 3) t
$$

(3)Avec ces hypothèses, la loi est tronquée dès que $\mu_{t}-3 \sigma_{t} \leq 0$. Il faut alors passer à une loi de type exponentielle. Le profil de corrosion initial (à 6 mois soit $\mathrm{t}=0$ ) sélectionné est présenté en figure 5 ; le point $\mathrm{A}$ est à $0 \mathrm{CM}$.
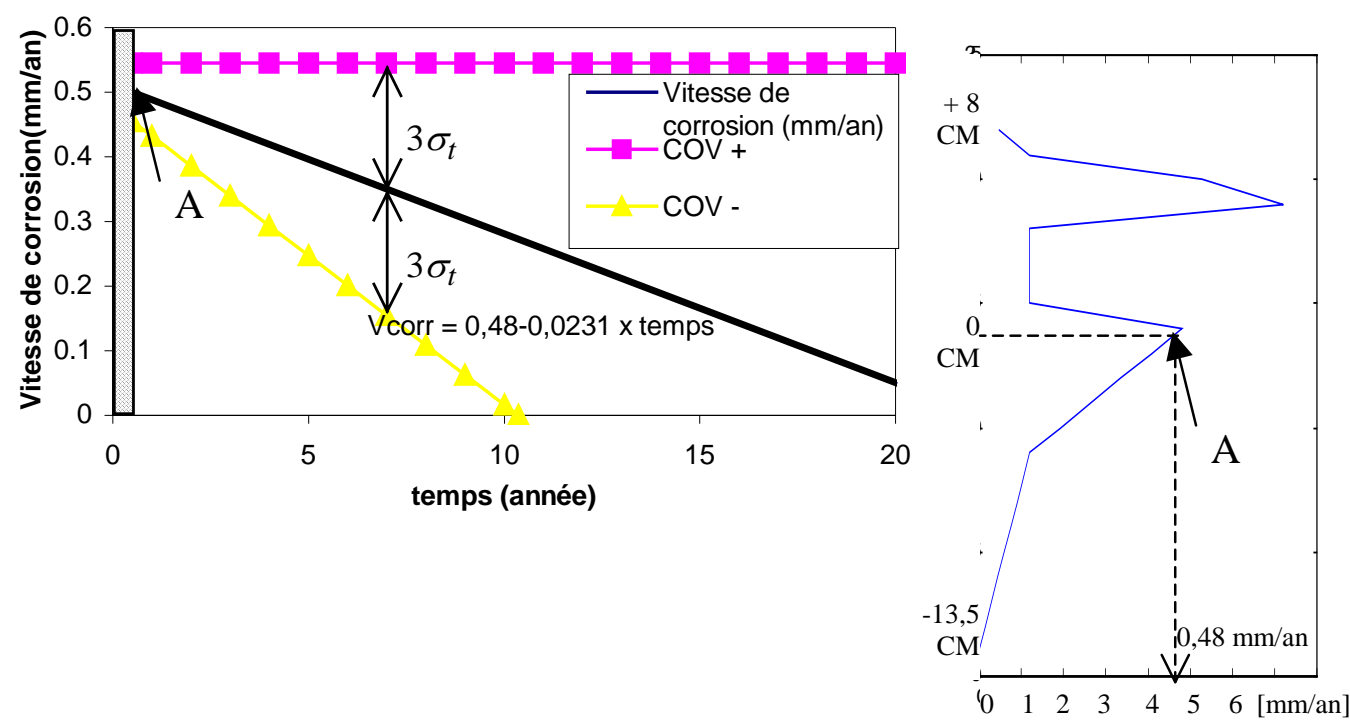

Figure 4: Evolution du processus moyen de corrosion à $0 m$ CM et des statistiques à +/- $3 \sigma$.

Figure 5: Profil moyen de vitesse de corrosion en fonction de la profondeur à $t=0$ (6mois).

\section{Evaluation des risques d'arrachement d'agrafes de palplanches}

Le critère d'état limite d'arrachement des agrafes en traction s’écrit (figure6) :

PxR-T $=0$

avec $\mathrm{T}$ résistance en traction, p poussée linéique du sol et $\mathrm{R}$ le rayon du gabion..

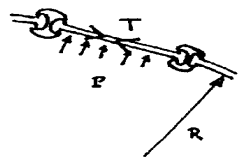

Figure 6: Grandeurs mécaniques pour le calcul d'agrafes 
Ici $\mathrm{R}$ et p sont des variables aléatoires. $\mathrm{T}$ est fonction de la section qui diminue avec le mécanisme de corrosion. Dans la suite, l'effort dû à la poussée a poussée est pris déterministe avec un coefficient de poussée Ka de 0.2 .

On suppose que la corrosion sur la face interne au gabion suit la même cinétique et le même profil que celui indiqué en figure 5.Pour le calcul, l'ouvrage est discrétisé en profondeur en tronçons de $1 \mathrm{~m}$ de hauteur. La probabilité de défaillance $\mathrm{P}_{\mathrm{d}}$ est alors calculée selon l'hypothèse que trois tronçons consécutifs $i-1$, i et $i+1$ sont défaillants soit en analyse système:

$\mathrm{P}_{\mathrm{d}}=\mathrm{P}\left(\mathrm{p}_{\mathrm{i}-1} \mathrm{R}-\mathrm{T}_{\mathrm{i}-1}<0\right) * \mathrm{P}\left(\mathrm{p}_{\mathrm{i}} \mathrm{R}-\mathrm{T}_{\mathrm{i}}<0\right) * \mathrm{P}\left(\mathrm{p}_{\mathrm{i}+1} \mathrm{R}-\mathrm{T}_{\mathrm{I}+1}<0\right)$

On peut ainsi envisager des ruines du type éventrement. La simulation est faite sous Matlab par la méthode de Monte-Carlo. Les évolutions temporelles sur 22 ans des probabilités sont représentées en figure 7. Elles correspondent aux six tronçons de 1m les plus profonds. Les probabilités système calculées par l'équation 4 sont présentées dans le tableau 2.

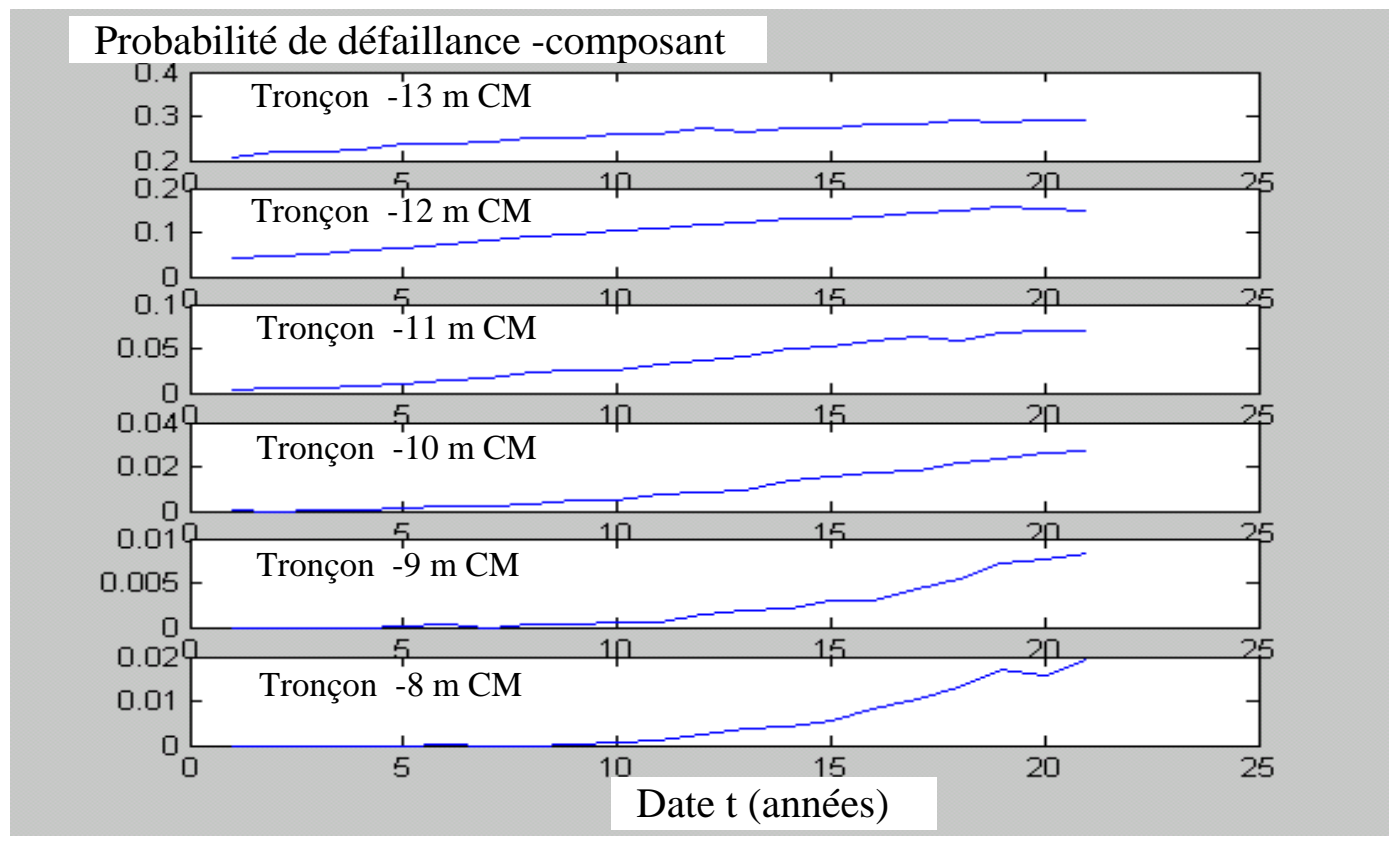

Figure 7: Evolution des probabilités de défaillance des 6 tronçons les plus profonds.

Tableau 2: Evolution de la probabilité de défaillance en fonction du temps.

\begin{tabular}{|c|c|c|c|c|c|c|c|c|}
\hline \multirow{2}{*}{$\begin{array}{l}\text { Profondeur du tronçon } \\
\text { médian (en CM) }\end{array}$} & \multicolumn{8}{|c|}{ Date (années) } \\
\hline & 0 & & 3 & 6 & 9 & 12 & 15 & 18 \\
\hline$-12 m$ & 0.0001 & 0.0001 & 0.0001 & 0.0002 & 0.0002 & 0.0003 & 0.0005 & \\
\hline$-10 m$ & 0 & 0 & 0 & 0 & 0 & 0 & 0 & \\
\hline$-8 m$ & 0 & 0 & 0 & 0 & 0 & 0 & 0 & \\
\hline
\end{tabular}

Au dessus de $-8 \mathrm{~m}$, les produits de probabilités ne sont pas reportés dans le tableau 2 car inférieurs à $10^{-5}$ et considérées comme nuls au vu des incertitudes sur les modèles ; l'incertitude sur les modèles, non-prise en compte ici donne à elle seule des probabilités de ruine de cet ordre de grandeur. 


\section{Conclusion}

Une étude de risque sur un quai en gabions a été réalisée à partir d'une méthode de sélection des états limites. Après avoir écarté les problèmes de vidange dus à une perforation, l'étude se focalise sur le dés-agrafage des palplanches par corrosion. On évalue la probabilité de défaillance selon un critère définit spécifiquement. Une cinématique de corrosion est présentée à cette fin, sous la forme d'un champ stochastique. On constate qu'en profondeur les probabilités sont non négligeables. Dans ce cas, le mécanisme de corrosion semble moins prépondérant que la poussée et une modélisation aléatoire de la poussée semble nécessaire.

\section{Références}

1.Labeyrie J., Schoefs F., "Matrix Response Surfaces For Describing Environmental Loads“, Vol. II Safety and Reliability, Proceeding of $15^{\text {th }}$ international conference on Offshore Mechanics and Arctic Engineering, (O.M.A.E'96), Florence, Italy, pp. 119-126 (ASME 1996).

2.Simon B., "Détermination des hauteurs d'eau extrêmes le long des côtes de France“, $4^{\text {èmes }}$ Journées Génie Civil - Génie Côtier, session II "aménagements estuairiens, télédétection, aménagement littoral", Dinard, pp. 167 - 175 (Ecole Pratique des Hautes Etudes Université du Havre, 1996).

3.Schoefs F., Rémond F., "Etude de risque appliquée à un quai en gabions soumis à de la corrosion“, Institut de Recherches en Génie Civil et Mécanique, Rapport interne n03-11-int02, nov. 2003, 89 pages.

4.ITASCA PFC2D (Particle Flow Code en 2 dimensions), version 3.0, Itasca consultant SA, 69130 ECULLY.

5.PLAXIS Finite Element Code for Soil and Rock Analyses, version 7.1.6.97

www.plaxis.com/ie.html

6.Masson S., Martinez J., Baylac B., Ferellec J.F., "Simulation numérique discrète des matériaux granulaires“ ,Mécaniques et Industries 4 (2003) 497-504.

7.Masson S., Martinez J., Mech. Cohes-Frict. Mater, "Multiscale simulations of the mechanical behaviour of an ensiled granular material“, 5 (2000) 425-442.

8.Memet J.B., "La corrosion marine des structures métalliques portuaires : études des mécanismes d'amorçage et de croissance des produits de corrosion“, Thèse de doctorat, Université de la Rochelle, (février 2000).

9.Aminot A., Chaussepied M., "Manuel des analyses chimiques en milieu marin“, IFREMER (1983).

10.Gounot A.M., Gaboriaud-Soubrier C., Quioc B., "Bio-corrosion dans les eaux douces“, Matériaux et techniques, numéro spécial bio-corrosion, pp 49-54,1990.

11.Tuyet Mait T.T., "Etude du comportement électrochimique d'un acier de construction navale dans l'eau de mer“, Thèse de doctorat, Institut National Polytechnique de Grenoble, (1988).

12.Melchers R.E, "Corrosion uncertainty modelling for steel structures", Journal of Constructional Steel Research, 52, pp3-19 Elsevier ed, 1999. 